\title{
Ferroelectric ordering and electroclinic effect in chiral smectic liquid crystals
}

\author{
Yu. G. Fokin 7 T. V. Murzina, and O. A. Aktsipetrov \\ Physics Department, Moscow State University, Moscow, 119992, Russia \\ S. Sorid and G. Marowsky \\ Laser-Laboratorium Goettingen e.V., Hans-Adolf-Krebs-Weg 1, D-37077 Goettingen, Germany
}

(Dated: October 30, 2018)

\begin{abstract}
Ferroelectric ordering, the electroclinic effect and chiral smectic C (SmC*) - smectic A (SmA*) phase transitions in thin planar ferroelectric liquid crystal (FLC) cells are studied by means of linear electrooptic and second harmonic generation techniques. The ferroelectric switching is detected in biased FLC cells by measuring azimuthal dependences of linear and nonlinear responses. The applied DC-electric field rotates the FLC symmetry axis with initial and final orientations in the cell plane. Comparative studies of the switching behavior in reflection and transmission allows to distinguish the contributions from the bulk and the sub-surface layers of the cell. The analysis of temperature dependence shows the existence of a strong surface coupling. The temperature dependent nonlinear polarization shows a critical behavior corresponding to the superfluid model.
\end{abstract}

PACS numbers: 64.70.Md, 42.65.Ky, 77.80.Fm, 61.30.Hn

\section{INTRODUCTION}

Ferroelectric liquid crystals (FLC) have been studied intensively for several decades. Chiral smectic liquid crystals (LC) have unique material properties like spontaneous polarization [1]. Although a single chiral smectic LC molecule has a non-zero dipole moment due to symmetry considerations 2], LC molecules in the bulk of the sample tend to form a helical structure which leads to polarization compensation. The helix can be unwound by application of a strong electric field or using thin test cells. Conventionally, alignment of LC is usually obtained by unidirectional mechanical rubbing of a thin polymer layer which coats the inner cell surfaces. Thus, the LC molecules point their long axes along the rubbing direction. This anisotropic interaction influences the ferroelectric ordering and the switching behavior which are of great practical importance for any LC device. The ferroelectrically ordered $\mathrm{SmC}^{*}$ phase is characterized by a non zero angle between the molecular long axis and the smectic layer normal. This tilt angle is the order parameter used to describe the second order $\mathrm{SmC}^{*}-\mathrm{SmA}^{*}$ phase transition.

In several studies the temperature dependence of the tilt angle below the critical temperature follows a power law of 0.5 (classical behavior) [3], whereas in other studies it follows a power law of 0.3 , in strong analogy with the superfluid helium predicted by de Gennes 41. The $\mathrm{SmA}^{*}$ phase in the vicinity of the transition point has also been the subject of intensive investigations, since

\footnotetext{
*Electronic address: yura@shg.ru URL: http://www.shg.ru

${ }^{\dagger}$ At present at ICFO-Institut de Ciencies Fotoniques, and Dept of Signal Theory and Communications, Universitat Politecnica de Catalunya, 08034 Barcelona, Spain
}

in that part of the critical region the electroclinic (EC) effect can induce a tilt angle which is proportional to the electric field strength [5]. The induced tilt angle is also strongly temperature dependent and diverges as the phase transition temperature is approached from above. Another type of this phenomenon, the so-called surface EC effect, is observed in the interfacial region of the FLC cells and originates from the interaction of the subsurface layers of chiral smectic molecules and a localized surface field [6]. In the vicinity of the transition point, the critical exponents of the tilt angle are in the range of $0.5 \div 1.5$ [4, 5, 6, 7]. The surface EC effect results in the existence of sub-surface region in which the director is twisted from the rubbing axis to the bulk alignment direction. The ferroelectric properties of these twisted layers are of great importance as they play a dominant role in LC-devices.

In this paper we use linear electrooptic (EO) and second harmonic generation (SHG) techniques for investigation of ferroelectric ordering, $\mathrm{SmC}^{*}-\mathrm{SmA}^{*}$ phase transitions and the EC effect in thin FLC cells. The main emphasis is made on comparative studies of the SHG reflection and transmission experimental geometries in order to figure out the roles of the sub-surface layers and the bulk in ferroelectric phase transitions. The SHG method is well-known for its sensitivity to symmetrical, structural and electronic properties of surfaces, interfaces and ultrathin films, and has been widely used along with EO for studying ferroelectric properties of chiral smectic LC [8]. As the SHG response strongly depends on the polar state of the matter because of its unique sensitivity to the breakdown of the inversion symmetry [9], it is a powerful instrument for probing ferroelectric phase transitions and electric-field induced effects in the vicinity of the critical region. 


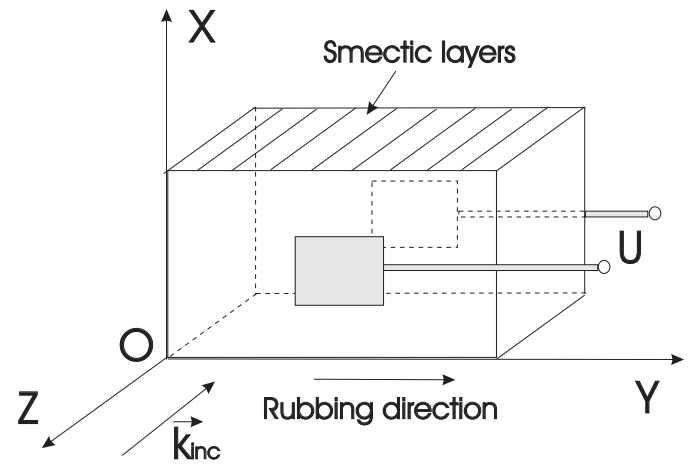

FIG. 1: Schematic representation of experimental geometry.

\section{MODEL DESCRIPTION}

We studied linear and nonlinear quadratic responses of the FLC cells upon application of an electric field and temperature variation up to the $\mathrm{SmC}^{*}-\mathrm{SmA}^{*}$ phase transition. Chiral smectic LC belong to the $C_{2}$ point group symmetry with the symmetry axes oriented in the cell plane. A schematic representation of the FLC cell and the coordinate system is shown in fig. 1 In this coordinate frame $O z$ is the normal to the cell, $X Y$ is the cell plane and the $C_{2}$ axis is parallel to the $O y$ direction and coincide with the main optical axis. We assume the FLC cell to be uniaxial, with the ordinary and extraordinary refractive indices $n_{0}=n_{x}=n_{z}$, and $n_{e}=n_{y}$, respectively. The transmittance of an optically active plate of the thickness $d$ and birefringence $\Delta n$ at the wavelength $\lambda$ in crossed-polarizers geometry is determined by the equation:

$$
T=\sin ^{2}(2 \alpha) \sin ^{2} \frac{\Delta n \pi d}{\lambda},
$$

where $\alpha$ is the angle between the main optical axes and polarization direction of the incident light. The linear electro-optic tensor $r_{i j k}$, where indices $i, j, k$ denote the axes of the cell coordinate system, for the given symmetry has 8 non-vanishing components $r_{x x y}, r_{y y y}, r_{z z y}, r_{y z x}$, $r_{y z z}, r_{x z y}, r_{x y x}, r_{x y z}$ [10. Application of the DC electric field $E$ along $O z$ direction leads to the rotation of the optical axes, which could be interpreted in terms of the rotation of the ellipse of refractive indexes. The equation of index ellipsoid in the presence of the electric field $E$ has the form

$$
\frac{x^{2}}{n_{x}^{2}}+\frac{y^{2}}{n_{y}^{2}}+\frac{z^{2}}{n_{z}^{2}}+2 y z r_{y z z} E+2 x y r_{x y z} E=1 .
$$

The transformation of (2) to the canonical form gives the DC field-induced rotation of the undisturbed system of coordinates based on the main optical axes. Considering the rotation only around $O z$-axis, the influence of the electric field can be described by the tilt of the main optical axis in the cell plane with the dependence of the turn angle $\Delta \alpha$ on the electric field as:

$$
\Delta \alpha=\frac{1}{2} \arctan \frac{2 r_{x y z} E}{\frac{1}{n_{o}^{2}}+\frac{1}{n_{e}^{2}}} .
$$

The nonlinear quadratic susceptibility tensor of the FLC cell with a $C_{2}$ symmetry is given by following nonvanishing components $\chi_{i j k}, \chi_{y x x}, \chi_{y y y}, \chi_{y z z}, \chi_{y z x}, \chi_{x y z}$, $\chi_{x x y}, \chi_{z y z}, \chi_{z x y}$ [11]. The contribution to the SHG intensity of each quadratic susceptibility component depends on the angle of incidence of the fundamental radiation, on the azimuthal position of the sample and polarizations of the input and SHG light. In transmission geometry at normal incidence only three components participate in SHG - $\chi_{y x x}, \chi_{x x y}$ and $\chi_{y y y}$, and for $p p$ geometry the SHG intensity can be expressed by:

$$
I_{p p}^{2 \omega} \sim \cos ^{2} \theta\left(\chi_{y y y} \cos ^{2} \theta+\left(2 \chi_{x x y}+\chi_{y x x}\right) \sin ^{2} \theta\right)^{2},
$$

where the azimuthal angle $\theta$ is the angle between the direction of the $p$-polarization and $C_{2}$ axes of the cell symmetry. By fitting the anisotropy dependences of the SHG intensity in all polarization combinations, the corresponding components of the quadratic susceptibility can be extracted.

According to a well-known model, in the smectic planar layers FLC molecules can precess on the surface of the smectic cone 12]. Ferroelectric switching is attributed to the interaction between molecular dipoles and external electric field and results in rotation of the molecules and, correspondingly, of the symmetry axes, within the half of the smectic cone. If the molecular director positions, corresponding to the saturating electric fields of opposite values, are in the cell plane and on the opposite sides of the smectic cone, then (3) can be used for the explanation of linear and nonlinear switching, as it shows the in-plane rotation of the main optical axis.

The angle between the molecular long axis and the normal to the smectic layers, i.e. the apex angle of the cone, is the order parameter of the $\mathrm{SmC}^{*}-\mathrm{SmA}^{*}$ phase transition, vanishing in the $\mathrm{SmA}^{*}$ phase. It can also have a finite magnitude in the $\mathrm{SmA}^{*}$ phase and diverge in the vicinity of the critical temperature due to the EC effect. The temperature-induced changes of this angle lead to the changes in $\alpha$ and $\theta$ angles which are determined by the orientation of the main axis. We show below that the temperature and electric field dependences of the main axis orientation can be deduced from linear and nonlinear optical experiments, in order to describe the ferroelectric switching, phase transitions and the EC effect in FLC cells.

\section{EXPERIMENT}

The principle arrangement of the experimental setup is described elsewhere [13]. Briefly, an OPO laser system is used as a source of the fundamental radiation, with the output wavelength of $537 \mathrm{~nm}$, repetition rate 
$10 \mathrm{~Hz}$ and pulse duration $4 \mathrm{~ns}$. An appropriate set of color filters is used for linear and nonlinear effects measurements. A photodiode or a PMT tube and gated electronics are used as a registration system for linear or nonlinear experiments. The temperature is varied from 20 to $55^{\circ} \mathrm{C}$, and automatically controlled by a digital thermocouple thermometer with the accuracy of $1^{\circ} \mathrm{C}$. An electric field in the range of -15 to $+15 \mathrm{MV} / \mathrm{m}$ is applied along $O Z$ axis through the ITO electrodes. Commercial FLC cells (E.H.C. Co., Tokyo) of a nominal thickness of $2 \mu m$ are used. These cells have an unidirectional rubbed polyimide surface layer. The samples are prepared by a slow cooling of the mixture [13], capillary filled in the isotropic phase. The critical temperature of the $\mathrm{SmC}^{*}$ $\mathrm{SmA}^{*}$ phase transition for the studied mixture is about $42^{\circ} \mathrm{C}$.

To determine the in-plane components of the nonlinear susceptibility, the SHG intensity vs. azimuthal angle is measured at normal incidence in transmission geometry for different polarization combinations - pp, ss, ps, $s p$, where the first letter denotes polarization of the incident light, and the second one - polarization of the light transmitted through the analyzer, placed after the sample. The observed anisotropy is shown in fig. [2 $\mathrm{k}, \mathrm{b}$ and correlates well with the dependence (4), obtained for a $C_{2}$ symmetry structure. No electric field is applied.

Ferroelectric switching of the cells is studied by measuring the anisotropy dependences of the SHG intensity at different values of the external DC electric field applied along the normal to the cell plane. Fig. 3a shows the SHG intensity in transmission geometry for $p p$-polarizations and for two applied DC fields of opposite sign. The dependences are shifted relative to each other, the phase difference is about $30^{\circ}$. In fig. 3 $\mathrm{b}$ the dependences of the SHG intensities vs. electric field are shown for different anisotropy positions of the cell. The anisotropy position determines the contrast value of the switching dependences and the ratio between SHG responses corresponding to opposite biases. Switching in reflection geometry also exhibits a shift of the anisotropy dependences for opposite electric fields with a phase difference of about $20^{\circ}$.

Linear transmittance anisotropy is measured in crossed polarizers geometry and is shown in fig. 2r. Application of the electric field leads to a shift of the linear transmittance anisotropy, as in the nonlinear case. In both cases, we observed a shift of about $30^{\circ}$.

Temperature dependences of the SHG response are taken in reflection geometry at incident angle about $45^{\circ}$ and in transmission at normal incidence. The cases of the presence and absence of external DC field are stud-

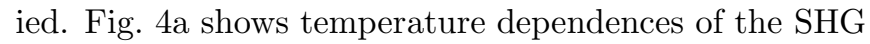
intensity in $p p$ transmission geometry for biased (filled circles and squares) and unbiased (open circles) cells. In the absence of the electric field the temperature dependence of the SHG intensity approaches a constant value at critical temperature in accordance with a power law at $T<T_{c}$, and shows no manifestation of the surface EC

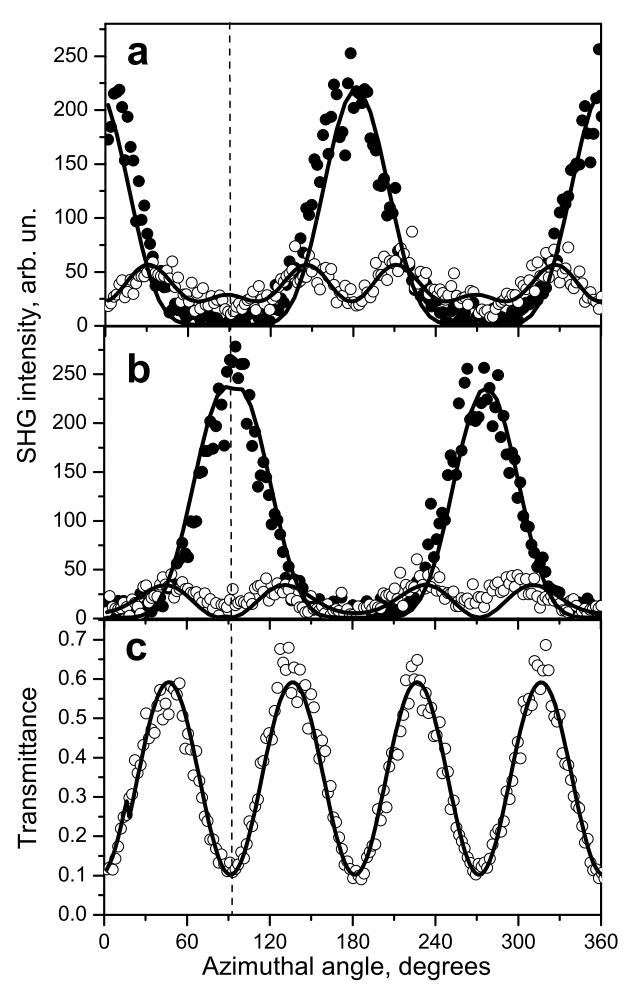

FIG. 2: Anisotropy dependence of the SHG intensity in transmission, a. ss (filled circles) and $s p$ (open circles) and b. $p p$ (filled circles) and ps (open circles) geometries; c. Anisotropy dependence of the linear transmittance at cross polarizers geometry. Solid lines are calculated from theory.

effect at $T>T_{c}$ in the SmA* phase. For biased LC samples, a critical behavior of the SHG intensity due to the EC effect is obtained in the $\mathrm{SmA}^{*}$ phase in the vicinity of the phase transition, which is seen from the divergent behavior of the SHG intensity at $T>T_{c}$, similar to the critical behavior of the tilt angle.

\section{DISCUSSION}

FLC cell structure usually contains layers with different direction of spontaneous polarization - twisted or helicoidally wounded layers. This modulation of the space orientation of molecular dipoles is governed by the intermolecular forces and substrate influence. We suppose that in the absence of the DC electric field the cell has a net dipole moment and axis of $C_{2}$ symmetry laying in the cell plane. Then it is possible to approximate anisotropy dependences of SHG intensity in all possible polarization combinations in transmission at normal incidence by formulas analogous to (4) for $p p$ case. In fig. 2] calculated curves are shown for all geometries. The approximation gives the ratios between in-plane components of nonlin- 


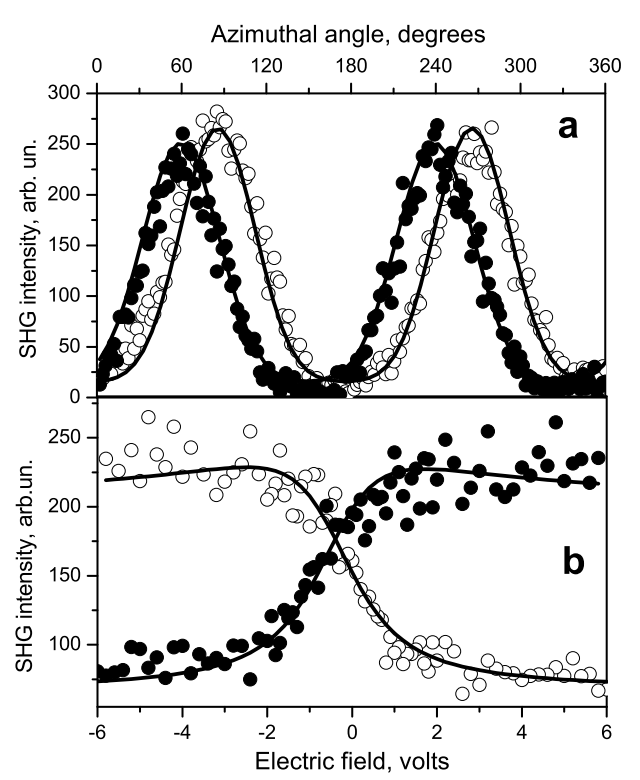

FIG. 3: a. Anisotropy dependence of the SHG intensity in transmission geometry at different electric field applied: $+8 \mathrm{~V}$ (filled circles), -8V (open circles). Solid lines are calculated by (14); b. SHG intensity dependence on the electric field in transmission for anisotropy positions 100 (open circles) and 230 (filled circles). Solid lines are calculated by (14) and (15).

ear susceptibility:

$$
\chi_{x x y}: \chi_{y y y}: \chi_{y x x}=1: 23.9:-3.3
$$

The linear transmittance anisotropy has four equal peaks and no dark extinction, which is typical for twisted structures. Assuming the main optical axis to lay in the cell plane, approximation of the linear anisotropy dependence by (11) gives a birefringence of $\sim 0.041$, which corresponds to the characteristic values for smectic LC measured by other authors 8]. Comparison of nonlinear and linear anisotropy dependences indicates that $C_{2}$ symmetry axis, corresponding to the maximum of the SHG anisotropy in $p p$-geometry, and the main optical axis, corresponding to the minimum of the linear transmittance anisotropy in $s p$-geometry, are parallel.

Temperature dependences of the SHG intensity for the applied electric field of different polarities reveal a hysteresis free $\mathrm{SmC}^{*}-\mathrm{SmA}^{*}$ second order phase transition. A typical critical behavior in the vicinity of the $\mathrm{SmC}^{*}$ $\mathrm{SmA}^{*}$ phase transition is obtained. A non-zero SHG signal in the $\mathrm{SmA}^{*}$ phase denotes that some part of the cell is still oriented and have a polar order with an in-plane component of the nonlinear polarization. The temperature dependence of the SHG response for reflection geometry shows qualitatively the same behavior as in transmission geometry, which is connected with the $\mathrm{SmC}^{*}$ $\mathrm{SmA}^{*}$ phase transition in the sub-surface layer of the cell. The SHG temperature dependence can be explained by the interference of the field and temperature dependent and independent contributions to nonlinear polarization, responsible for the SHG signal. Then, we can write for the nonlinear polarization

$$
\vec{P}_{N L}^{2 \omega}=\vec{P}_{\text {surf }}^{2 \omega}(E, T)+\vec{P}_{\text {bulk }}^{2 \omega}(E, T)+\vec{P}_{\text {const }}^{2 \omega}
$$

where $\vec{P}_{\text {surf }}^{2 \omega}(E, T)$ and $\vec{P}_{\text {bulk }}^{2 \omega}(E, T)$ are electric field $E$ and temperature $T$ dependent contributions to the nonlinear polarization at double frequency from the subsurface layers and the bulk, respectively, and $\vec{P}_{\text {const }}^{2 \omega}$ is a field and temperature independent component. For the transmitted SHG the correlation length is about 5 microns, while for reflection it is about 0.1 micron [14], which indicates that in reflection a thin sub-surface layer participates in SHG. As SHG temperature dependences of sub-surface layers and the bulk have the same qualitative character, then either $\vec{P}_{\text {bulk }}^{2 \omega}(E, T)$ and $\vec{P}_{\text {surf }}^{2 \omega}(E, T)$ from (6) should be the same functions of $E$ and $T$, or the bulk contribution to nonlinear polarization should vanish.

Explanation of temperature dependences demands the existence of an electric field and temperature independent in-plane contribution $\vec{P}_{\text {const }}^{2 \omega}$ to the nonlinear polarization. Orientation of the molecules in the bulk layers of the cell can be electric-field independent in some experimental geometries depending on the layer packing, but temperature dependence is obligatory because of the presence of the $\mathrm{SmC}^{*}$-SmA* phase transition. Then the non-switching layer should be in the sub-surface region. We can assume that a "frozen" sub-surface layer, with a thickness smaller than the correlation length for SHG in reflection geometry, exists. It is strongly stabilized by the surface coupling, and does not respond to any external electric field or temperature variations, as well as the layer closest to the substrate. The latter has only $z$-component of the nonlinear polarization, as the dipole moments of its molecules are directed along the normal to the substrate plane, and then it does not contribute to the SHG at normal incidence. But the rest of the "frozen" region contains layers with in-plane components of spontaneous polarization due to the twist near the surface, and thus participates in the SHG at any angle of incidence.

Let us figure out the interconnection between the temperature dependences of SHG and the order parameter of the $\mathrm{SmC}^{*}-\mathrm{SmA}^{*}$ phase transition. Usually the order parameter of this phase transition is the tilt angle, as spontaneous polarization is compensated because of the helical structure. The thickness of the cells studied in this paper is smaller than the helix pitch, besides, anisotropic interaction with the substrate produces an additional orientational order of the FLC molecules, so that the cells have spontaneous polarization $\vec{P}_{s p}$ in $\mathrm{SmC}^{*}$ phase. $\vec{P}_{s p}$ decreases in $\mathrm{SmA}^{*}$ phase and is a linear function of the tilt angle $\theta(E, T)$. So we can write for the SHG intensity:

$$
\sqrt{I^{2 \omega}} \sim \vec{P}_{N L}^{2 \omega} \sim \vec{P}_{s p} \sim \theta(E, T)
$$


assuming that $\sqrt{I^{2 \omega}(E, T)}$ has the same critical dependence on temperature as the tilt angle. Then, according to (6) and (7), the temperature dependent contribution $\sqrt{I^{2 \omega}(E, T)}$ in the absence of the electric field and for $T<T_{c}$ can be approximated by the following expression:

$$
\sqrt{I^{2 \omega}(E=0)} \sim P_{\text {const }}^{2 \omega}-P_{0}^{2 \omega}\left(1-\frac{T}{T_{c}}\right)^{\beta},
$$

where $P_{c o n s t}^{2 \omega}$ and $P_{0}^{2 \omega}$ are proportional to isotropic and anisotropic contributions of nonlinear polarizations, $T_{c}$ is the temperature of the phase transition, and $\beta$ is the critical exponent. Approximation of the curve is shown in fig. $4 \mathrm{~h}$ by a solid line, with $\beta=0.31$, in correspondence to the theory of phase transition in a superfluid helium.

To compare the temperature behavior of the nonlinear polarization in the vicinity of the phase transition for reflection and transmission cases, we introduce the contrast of the dependences as

$$
K=\frac{\sqrt{I^{2 \omega}(+E)}-\sqrt{I^{2 \omega}(-E)}}{\sqrt{I^{2 \omega}(+E)}+\sqrt{I^{2 \omega}(-E)}}
$$

where $I^{2 \omega}( \pm E)$ are the SHG intensities for positive and negative voltages applied to the cell. In fig. 4p the contrast vs. temperature is shown for reflection and transmission geometries. The contrast determined in such a way shows a behavior similar to that of the order parameter near the phase transition. Introducing SHG intensity dependences on temperature in the presence of the electric field at $T<T_{c}$ in the way, analogous to (8),

$$
\sqrt{I^{2 \omega}( \pm E)} \sim P_{\text {const }}^{2 \omega} \pm P_{0}^{2 \omega}( \pm E)\left(1-\frac{T}{T_{c}}\right)^{\beta},
$$

and supposing $P_{0}^{2 \omega}(E)=P_{0}^{2 \omega}(-E)$, we can express the contrast $K$ as:

$$
K \sim \frac{P_{0}^{2 \omega}(E)}{P_{\text {const }}^{2 \omega}}\left(1-\frac{T}{T_{c}}\right)^{\beta} .
$$

Approximation of experimental dependences by (11) in

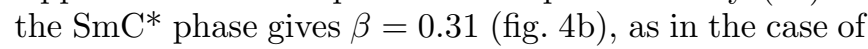
the absence of the field. The amplitude of the contrast (11) depends on the ratio $P_{0}^{2 \omega}(E) / P_{\text {const }}^{2 \omega}$ of field dependent and independent contributions to the nonlinear polarization. In transmission this ratio can be bigger due to the larger amount of layers participating in ferroelectric switching, besides, the field independent contribution of the boundary layer leads to the contrast decrease in reflection geometry. These considerations explain why the contrast of temperature dependences and electric fieldinduced shift of anisotropy dependences in transmission are bigger than in reflection geometry, as obtained in the experiment.

Temperature dependence of the contrast in the region of the $\mathrm{EC}$ effect, at $T>T_{c}$, can be written in the same way, considering the temperature dependence

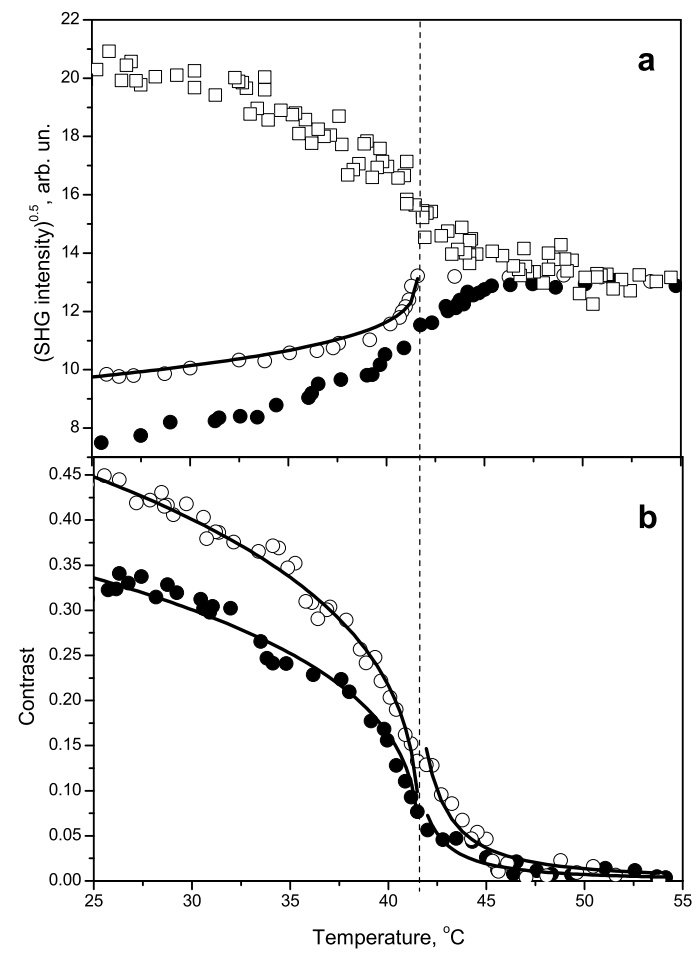

FIG. 4: a. Temperature dependence of the SHG intensity in transmission geometry at different applied electric field : $+8 \mathrm{~V}$ (squares), 0V (open circles), $-8 \mathrm{~V}$ (filled circles). Solid line is a fit by (8); b. Temperature dependence of the contrast in reflection (filled circles) and transmission (open circles) geometries. Solid lines are calculated by (11) and (13).

$\sqrt{I^{2 \omega}(E, T)}$ similar to the temperature dependence of the tilt angle above the critical temperature:

$$
\sqrt{I^{2 \omega}( \pm E)} \sim P_{\text {const }}^{2 \omega} \pm P_{0}^{2 \omega}( \pm E)\left(1-\frac{T}{T_{c}}\right)^{-\gamma},
$$

where $\gamma$ is the critical exponent, which gives under the same considerations the contrast for $T>T_{c}$ :

$$
K \sim \frac{P_{0}^{2 \omega}(E)}{P_{\text {const }}^{2 \omega}}\left(1-\frac{T}{T_{c}}\right)^{-\gamma},
$$

Approximation of experimental data (fig. 40) gives $\gamma=$ 1.4. Thus we observe that both critical exponents are in agreement with the superfluid helium model.

Application of a DC electric field to the FLC leads to a shift of anisotropy dependences of the linear and nonlinear responses, as shown in fig. 3 a. Similar pictures are obtained in the reflection case. As the character of anisotropy dependences is the same in the presence of the field, our suggestion of the switching mechanism based on the symmetry axis rotation in the cell plane seems to be rather realistic. The shift originates from the rotation of the molecules in the switching layers, the resulting nonlinear polarization of the cell is the sum of unchanged quadratic polarization from the "frozen" layer and from field-dependent region with the new position of the symmetry axes. Thus for approximating the SHG anisotropy 
dependence in the presence of the electric field, we divide the nonlinear polarization into two parts, and (4) can be rewritten as:

$$
I_{p p}^{2 \omega} \sim\left(g P_{N L, p p}^{2 \omega}(\theta+d \theta)+(1-g) P_{N L, p p}^{2 \omega}(\theta)\right)^{2},
$$

where $g$ is the effective thickness of switching layer relatively to the non-switching one and $d \theta$ is a field-induced change of the azimuthal angle. The value of $g$ is estimated to be 0.5 which indicates that the magnitude of the field-independent contribution is comparable to the fielddependent one, allowing effective mutual interference in accordance with (6). Symmetry axis shift $d \theta$ between opposite values of the voltage is about $52^{\circ}$. For electric field dependences of the SHG intensity, shown in fig. $3 \mathrm{~b}$, the independent variable is the electric field and in (14) the azimuthal angle $\theta$ is fixed by the anisotropy position. Then $d \theta$ becomes a function of the electric field, chosen, according to (3), as a saturation function:

$$
d \theta(E)=b \arctan (c(E-d E)),
$$

where $b$ and $c$ are constants, depending on the strength of interaction between field and angle, and $d E$ is a constant determined by the switching history, as ferroelectric switching of SHG intensity exhibits characteristic field hysteresis. Experimental dependences in fig. 30 are taken for two anisotropy positions with the difference in $\theta \sim 130$ degrees. Fitting one of the curves by (14) with (15) allows to extract $b=0.5$ and $c=0.63$. Varying then only $d E$ and $\theta$ during approximation of the second curve gives the difference of the anisotropy position of about 126 degrees, in good agreement with experimental conditions. Values of $d E$ in both cases lay in the region of typical hysteresis width and do not exceed $1 \mathrm{~V} /$ micron. The value of $b$ corresponds to the tilt of the symmetry axis in the cell plane, discussed above (3).

In conclusion, the electroclinic effect, the $\mathrm{SmC}^{*}-\mathrm{SmA}^{*}$ phase transition and ferroelectric switching have been studied in thin planar cells of ferroelectric chiral liquid crystals by means of electrooptic and second harmonic generation techniques. The analysis of the temperature dependences of nonlinear quadratic response in the critical region leads to the assumption of a strong surface coupling existence, resulting in the stabilizing of several "frozen" subsurface twisted layers, independent on electric field and temperature. Critical exponents in the vicinity of the $\mathrm{SmC}^{*}{ }_{-} \mathrm{SmA}{ }^{*}$ phase transition show a behavior corresponding to the superfluid helium theory. Ferroelectric switching has been observed in linear and nonlinear anisotropic responses and has been explained by the model, assuming in-plane rotation of symmetry and main optical axes of the cells in the presence of external electric field.

\section{Acknowledgments}

We thank Prof. G. Heppke's group for synthesis of the samples used in our work. The work was supported by INTAS grant 2002-113/F1b, President grant "Leading Russian Scientific Schools" 1604.2003.2 and EU network grant ERB FMRXCT 980209 (SILC). S.S acknowledges funding from European Regional Development Fund and from the Ministerio de Ciencia y Tecnologia through the Ramon y Cajal program.
[1] R.B. Meyer, L. Liebert, L. Strzelecki, P. Keller, J. Phys. (Paris) 36, L69 (1975).

[2] R.B. Meyer, Mol. Crys. Liq. Crys. 40, 33 (1977).

[3] I. Musevic, R. Blinc, B. Zeks, The Physics of Ferroelectric and Antiferroelectric Liquid Crystals (World Scientific, Singapour, 2000).

[4] P.G. de Gennes and J. Prost The Physics of Liquid Crystals (Oxford University Press, New York, 1993); E. Olbrich, O. Marinov, and D. Davidov, Phys. Rev. E 48, 2713 (1993).

[5] S. Garoff and R.B. Meyer, Phys. Rev. Lett. 38, 848 (1977); S. Garoff and R.B. Meyer, Phys. Rev. A 19, 338 (1979).

[6] W. Chen, Y. Ouchi, T. Moses, Y.R. Shen, K.H. Yang, Phys. Rev. Lett. 68, 1547 (1992); Jiuzhi Xue and Noel A. Clark, Phys. Rev. Lett. 64, 307 (1990).

[7] Jonathan V. Selinger, Peter J. Collings, and R. Shashidhar, Phys. Rev. E. 64, 061705 (2002).

[8] M. Loddoch, G. Marowsky, H. Schmid, G. Heppke, Appl.
Phys. B 59, 591 (1994); Byoungchoo Park, San-seong Seomun, Michi Nakata, Masayoshi Takanashi, Yoichi Takanishi, Ken Ishikawa and Hideo Takezoe, Jpn. J. Appl. Phys. 38, 1474 (1999); N. Pereda, J. Ortega, C.L. Folcia, and J. Etxebarria, Phys. Rev. E. 62, 2334 (2000).

[9] N. Blombergen, R.K. Chang, S.S. Jha, C.H. Lee, Phys. Rev. 174, 813 (1968).

[10] Yariv and P.Yeh, Optical Waves in Crystals (John Wiley and Sons, New York 1983).

[11] Y.R. Shen, The principles of Nonlinear Optics (John Wiley and Sons, New York 1984).

[12] Byoungchoo Park, Michi Nakata, San-seong Seomun, Yoichi Takanishi, Ken Ishikawa, and Hideo Takezoe, Phys. Rev. E. 59, R3815 (1999).

[13] Yu.G. Fokin, T.V. Murzina, O.A. Aktsipetrov, S. Soria, G. Marowsky, Surf. Sci. 507, 724 (2002).

[14] Y.G. Fokin, T.V. Murzina, O.A. Aktsipetrov, S. Soria, G. Marowsky, Appl. Phys.B 74, 777 (2002). 